\title{
La socialidad humana como anomalía evolutiva
}

\author{
Pablo N avarro \\ Universidad de 0 viedo \\ pnavarro@correo.uniovi.es
}

\section{Resumen}

La teoría sociológica ha pretendido captar el complejo fenómeno de la socialidad humana por medio de conceptos tales como la distinción micro/macro, la relación entre acción y estructura o la contraposición entre el enfoque individualista y el holista. El objetivo de este artículo es mostrar de qué manera esas aproximaciones conceptuales pueden resultar clarificadas, y encontrar una raíz común, apelando al proceso evolutivo que ha configurado la forma de socialidad propia de nuestra especie.

A este respecto, la socialidad humana parece fundarse en una peculiar dobleduplicidad. Ésta constituye el fundamento de las dualidades conceptuales indicadas, que han aguijoneado buena parte de las reflexiones teóricas de la sociología desde sus comienzos.

Palabras clave: mapas sociocognitivos, inteligencia colectiva, sujeto social, socialidad humana, socialidad animal, teoría sociológica, sociobiología.

\begin{abstract}
Sociological theory has attempted to grasp the complex phenomenon of human sociality by means of concepts such as the micro/macro distinction, the relation between action and structure, or the clash between the individualistic and the holistic stances. The aim of this paper is to show how those conceptual approaches get some clarification, and find a common underpinning, when we look into the evolutionary process that shaped the sociality of our species.

In this respect, human sociality seems to hinge on a peculiar double duplicity. This provides the basis for the aforementioned conceptual dualities, which have prompted a substantial part of the theoretical gogitations of sociology from its beginings.
\end{abstract}

Key words: sociocognitive maps, collective intelligence, social subject, human sociality, animal sociality, sociological theory, sociobiology.

\section{Sumario}

1. La doble duplicidad de la socialidad humana, según la teoría social

2. La doble duplicidad de la socialidad humana como dispositivo sociogenético

3. Los dos caminos evolutivos de la socialidad animal

4. Las sociedades animales y sus dos tipos de mapas sociocognitivos: externos e internos
5. La condición anómala de la socialidad humana

6. Conclusiones: entorno natural socializado, entorno social naturalizado, y vuelta a la doble duplicidad

Bibliografía 


\section{La doble duplicidad de la socialidad humana, según la teoría social}

$\mathrm{H}$ ay varias formas de describir las perplejidades de fondo que suscita el complejo fenómeno de la socialidad humana. Así, son muchos los autores que se refieren a la brecha «micro/macro»; ${ }^{1}$ otros apuntan a la relación dialéctica entre «acción»y «estructura», ${ }^{2}$ a las diferencias entre el enfoque «holista» y el «individualista», ${ }^{3} 0$ a la distinción entre «objetividad social» $\mathrm{y}$ «subjetividad social». ${ }^{4}$ Todas estas perspectivas arrojan luz sobre aspectos importantes del complejo fenómeno de la socialidad humana, si bien parecen hacerlo de manera un tanto unilateral - se trata de puntos de vista que, claramente, se cruzan sin lograr integrarse.

En este artículo voy a proponer una aproximación relativamente heterodoxa a algunas de las cuestiones más básicas de la teoría sociológica. Y voy a hacerlo por medio de una distinción conceptual diferente de las anteriormente mencionadas: la distinción a la que llamaré la doble duplicidad de la socialidad humana. En realidad, se trata de una idea que ha estado presente, de manera más bien implícita, a lo largo de todo el desarrollo de nuestra disciplina. En lo que sigue, intentaré elaborar explícitamente esta distinción largo tiempo soterrada, y trataré de mostrar, por una parte, cómo la misma puede actuar en calidad de puente entre enfoques teóricos diferentes e incluso opuestos. Y, por otra, de qué modo la distinción en cuestión se corresponde con un rasgo básico de nuestra peculiar evolución como especie biológica. Este último hecho debería suministrar una dosis adicional de credibilidad ontológica a la propuesta conceptual que me dispongo a exponer.

La idea que subyace a la expresión «la doble duplicidad de la socialidad humana» es, de entrada, simple. En la socialidad humana, es decir, en el conjunto de capacidades que permiten a los seres humanos constituir sociedad (alguno de los muy variados tipos de sociedad que somos capaces de edificar los individuos de nuestra especie), cabe distinguir dos niveles de duplicidad. El primero de esos niveles se corresponde con la duplicidad que llamaré primaria; el segundo, con la duplicidad que denominaré secundaria. La duplicidad primaria apunta a la distinción - y a la interacción conflictiva - entre el individuo «presocializado» y el «socializado» - entre nuestro I y nuestro me, para expresarlo en los términos de M ead. ${ }^{5}$ La duplicidad secundaria se refiere a la distinción, y a la compleja interacción, entre nuestro self socializado - el cual incluiría la primera duplicidad, ésa que relaciona y a la vez enfrenta I y me- , y nuestro entorno social objetivo. Este entorno, como veremos, es un resultado emergente de procesos que sólo en parte son de naturaleza intencional.

1. Lam O de Espin OSA, E. [1996]; Alexander, J. C. y otros (eds.) [1987]; H uber, J. [1991]; SCHELLING, T. C. [1978].

2. Giddens, A. [1986]; AleXANDer, J. C. [1990].

3. Coleman, J. S. [1994 ]; Elster, J. [1986]; Phillips, D. C. [1976].

4. Durkheim, É. [1987]; LuHMAN N, N. [1998]; N AVARRo, P. [1997].

5. MEAD, G.H. [1967]. 
La duplicidad primaria ha sido tratada por muchos autores, de $\mathrm{G}$ eorge $\mathrm{H}$. M ead a Tal cott Parsons. ${ }^{6}$ Teorías sociológicas básicas, como la de la social ización, fueron diseñadas para sondear y explicar esta duplicidad, que se revela a través de la relación, a la vez conflictiva y creativa, entre el individuo social y su entorno cultural inmediato. Esta duplicidad primaria está presente - si bien de manera idiosincrásica- en todas las sociedades humanas, desde las culturas más antiguas desarrolladas por el H omo sapiens, hasta nuestras sofisticadas sociedades posfreudianas. Se trata de una duplicidad congénita a nuestra especie, y que deberá persistir en cualquier sociedad reconocible como humana.

La duplicidad secundaria comenzó a sernos revelada, principalmente, por autores clásicos como Adam Smith y Karl M arx. En realidad, Adam Smith nos proporciona, a este respecto, dos retratos conceptuales alternativos de la socialidad humana; el que traza en su «Teoría de los sentimientos morales», ${ }^{7}$ y el que bosqueja en «L a riqueza de las naciones». ${ }^{8}$ En la primera obra, Smith explica cómo la capacidad del individuo humano para establecer vínculos sociales con otros individuos congéneres, se fundamenta en dispositivos como la simpatía y la reflexión consciente. Es justamente esta capacidad - y volveremos sobre ello- la que permite el desarrollo de lo que he denominado la «duplicidad primaria». En la segunda obra, por el contrario, Smith se refiere a un tipo muy diferente de vínculo social, establecido por medio de relaciones de mercado regidas en exclusiva por el interés de los agentes económicos. Esas relaciones prosperan gracias a la división del trabajo, al tiempo que la fomentan. Este tipo de dinámica social es el que daría forma a lo que denomino la «duplicidad secundaria» de la socialidad humana.

Karl M ar ${ }^{9}$ contempla el hecho de la socialidad a través de la lente que le suministra la producción material. Para este autor, el desarrollo de las fuerzas productivas ha conducido a una contradicción fundamental entre los requerimientos básicos de la naturaleza humana y las circunstancias de alienación impuestas por la economía capitalista. Estas circunstancias de alienación (o de explotación, si se prefiere) conducen a la emergencia de un entorno social objetivo sobre el cual los individuos carecen de control. Este entorno, definido por el fenómeno de la reificación, está claramente emparentado con el vínculo social no intencional que Smith analizó, y se hallaría asimismo en el origen de esa duplicidad secundaria que resulta típica de las sociedades modernas.

\section{La doble duplicidad de la socialidad humana como dispositivo sociogenético}

No voy a intentar dar aquí una explicación detallada de la dinámica generada por esta doble duplicidad, cuyos perfiles conceptuales pretendo simplemente
6. PARSONS, T. [1964]
7. SMITH, A. [1984].
8. SмITH, A. [1991].
9. M ARX, K. [1973]. 
bosquejar. Baste decir que, desde la perspectiva que proporciona la tesis de la doble duplicidad, el agente individual se encuentra, por un lado, permanentemente situado en la inestable frontera que separa - pero al propio tiempo relaciona - I y me (es decir, en la duplicidad de la primera clase). M as, por otro, ese mismo agente, como totalidad que integraría esa duplicidad I/me, se halla a su vez sometido a las tensiones que le impone vivir en una segunda frontera: la que lo separa y al mismo tiempo lo relaciona dinámicamente con su entorno social objetivo (ésta sería la duplicidad de la segunda clase). Así, la duplicidad primaria anida dentro de la secundaria, y ésta a su vez resulta reflejada (en forma de imágenes de conciencia) por la primera. El proceso de la morfogénesis social sería, al menos en el nivel del agente individual, el resultado del cruce creativo de esas dos fronteras. Los flujos en la primera frontera producen cambios «culturales» - de hábitos, actitudes y creencias, en el sentido orteguiano del término. ${ }^{10} \mathrm{El}$ cruce de la segunda frontera produce cambios socio-políticos, a través de aparatos cognitivos (ciencia y conocimiento experto) y reguladores (Estado, política, organizaciones...) de carácter especializado.

Si estoy dando beligerancia teórica a esta idea de la doble duplicidad es, en primer lugar, porque la misma podría ser capaz de acomodar, dentro de una perspectiva unitaria, algunas distinciones firmemente arraigadas en el campo de la teoría sociológica. M e refiero a parejas conceptuales como la distinción macro/micro, la que contrapone estructura y acción, la que media entre el enfoque individualista y el holista, la que suele trazarse entre la objetividad y la subjetividad social, la que se ha propuesto establecer entre sociedad y cultura, ${ }_{11}^{11}$ o la postulada entre deseos e intereses. ${ }^{12}$ Esa acomodación, no obstante, exigirá afinar cada una de estas dicotomías, mostrando cómo se cruzan y se reflejan las unas en las otras.

Así, por ejemplo, lo que podría concebirse como «subjetividad social micro» (digamos, las regularidades sociales «etnometodológicamente» asumidas por el individuo) pertenecería, por una parte, a la duplicidad primaria. Es esta duplicidad, precisamente, la que configura al sujeto socializado (al me), en su relación a un tiempo conflictiva y productiva con el I. Pero, por otra parte, esa «subjetividad social micro» pertenecería asimismo a la duplicidad secundaria. Pues incluiría la conciencia (siempre imprecisa y limitada) que los individuos desarrollan acerca de las reglas que regulan, no sólo sus tratos con otros individuos, sino también la «objetividad social» que constriñe su acción. Esta «objetividad social», como se ha sugerido, se constituye a partir de los efectos reificadores que la acción humana tiende a generar en sociedades hipercomplejas como las modernas. 
D e manera análoga, lo que puede contemplarse como la «objetividad micro social» (por ejemplo, los productos disponibles para el consumidor en un determinado mercado) sería un fenómeno concomitante, por un lado, con la duplicidad primaria (al asociarse, digamos, a las «pulsiones adquisitivas» del individuo, que expresarían la relación a menudo conflictiva entre el patrón cultural por él asumido y sus preferencias estrictamente personales). Pero, por otro lado, esa «objetividad micro social» involucraría asimismo la duplicidad secundaria. Y ello en la medida en que, para seguir con el ejemplo, los precios de esos productos disponibles en el mercado encarnan y generan ciertas constricciones objetivas. En concreto, y por citar sólo una de ellas, la que cierta estructura de precios, unida al nivel de ingresos de cada consumidor - realidades determinadas más que nada por procesos «macro»- impone sobre los patrones culturales y personales que modelan el consumo de los individuos.

O bsérvese, a este respecto, un hecho destacable. En las sociedades humanas más simples la mayor parte de la «objetividad micro» que los individuos enfrentan es de natural eza no social - resulta impuesta directamente por las condiciones ecosistémicas naturales. Por el contrario, en los entornos sociales más complejos que nuestra especie ha edificado a lo largo de los últimos siglos, ocurre a la inversa: la mayor parte de la «objetividad micro» que afrontan los agentes es un constructo social - es resultado de procesos sociales de largo alcance, sólo parcialmente intencionales, a través de los que se actualizan los efectos reificadores de la acción humana. Esos efectos engendran un entorno social fuertemente cosificado, capaz de constreñir de forma eficaz y consistente la acción del individuo.

La propuesta de utilizar la idea de la doble duplicidad como eje estructurador de los conceptos más básicos de la teoría sociológica parecerá tal vez caprichosa, 0 al menos excesiva. $\mathrm{H}$ ay un hecho, sin embargo, que concede un respaldo ontológico de singular peso a esta propuesta. Este hecho es la clara sintonía de la misma con nuestros conocimientos actuales acerca de la evolución, tanto de la socialidad humana como de la socialidad animal. Como intentaré mostrar en las páginas que siguen, lo que he denominado la «duplicidad primaria» es un fenómeno emergente de la evolución biológica. La duplicidad secundaria, por el contrario, es un fenómeno emergente de la evolución cultural.

En las secciones que restan intentaré, pues, avanzar en la clarificación de la doble duplicidad que nos ocupa. Y lo haré, en primer lugar, por medio de la traducción de la misma al lenguaje de la evolución biológica. Esta traducción evidenciará el profundo arraigo etológico de la duplicidad en cuestión, y nos ayudará a entender la anomalía que la socialidad humana representa dentro del cuadro general de la socialidad animal. Esta anomalía constituye la diferencia fundamental entre las sociedades humanas y las demás sociedades animales. Sólo en las primeras, como veremos, se despliega la forma sui generis de complejidad social que se encarna en la evolución cultural, y se realiza a través de la experiencia irrepetible de la historia. 


\section{Los dos caminos evolutivos de la socialidad animal}

Cuando se contempla la evolución de la socialidad animal en su conjunto, ${ }^{13}$ nos topamos con una aparente paradoja: al gunos animales que, de acuerdo con nuestros criterios más o menos intuitivos, consideraríamos relativamente estúpidos, son capaces de constituir sociedades a las que la mayoría de nosotros atribuiríamos un al to grado de complejidad; mientras que animales que pare cen ser mucho más inteligentes que los anteriores sólo están facultados, a primera vista, para en samblar sociedades relativamente simples. Por ejemplo, las hormigas y otros insectos eusociales son capaces de poner en pie sociedades mucho más elaboradas, en apariencia, que las constituidas por lobos o gorilas. O bsérvese, a este respecto, que las sociedades establecidas por los insectos sociales suelen ser no sólo más numerosas que las de los mamíferos. También exhiben una estructura interna mucho más minuciosa y trabada que las de otras sociedades animales más cercanas a nosotros en términos evolutivos.

Esta aparente paradoja bien pudiera ser el punto de partida de una incursión teórica productiva para la teoría social. Con este propósito en mente, abordaremos el desafío intel ectual que representa este desajuste entre «grado de inteligencia individual» $\mathrm{y}$ «grado de complejidad social». Y lo haremos tratando de fijar las diferencias entre los distintos tipos de socialidad involucrados en cada caso. C onviene puntualizar, a este respecto, que cabría definir la socialidad de una determinada especie animal como el conjunto de dispositivos que instrumentan la efectiva sincronización entre las conductas de los individuos que componen las sociedades constituidas de manera viable por esa especie. Esos dispositivos de sincronización conductual pueden concebirse - si damos un sentido lo suficientemente amplio a nuestra idea de cognición- como mecanis mos sociocognitivos. El lenguaje verbal es, claramente, un mecanismo sociocognitivo peculiar de nuestra especie. Pero otros sistemas productores de señales - más simples, y de natural eza visual o bioquímica- suelen funcionar también, en otras especies animales, como poderosos mecanismos sociocognitivos, capaces de instrumentar esos procesos de sincronización conductual que constituyen el modus operandi de cualquier sociedad animal.

Algunos autores ${ }^{14}$ han propuesto distinguir entre varios tipos de estrategias evolutivas presentes en el curso del desarrollo de la socialidad animal. Esas distintas estrategias se fundamentarían en el despliegue de mecanismos sociocognitivos de distinta índole. Simplificando al máximo la cuestión, puede afirmarse que el proceso de desarrollo de la socialidad animal ha seguido dos estrategias principales en su despliegue evolutivo. Como veremos, esas dos estrategias el aboran sus correspondientes mecanismos sociocognitivos en direcciones opuestas, y están representadas por los dos grupos de animales ya mencionados - los insectos eusociales y los mamíferos. Estos dos distantes taxa encarnan los dos pináculos evolutivos de la socialidad animal. Aunque pueda parecer extraño,

13. WILSON, E. O. [1980].

14. Chialvo, D. R. y M ILLonas, M . M . [1995]. 
la comparación entre los mecanismos de socialidad que son típicos de esos dos grupos tan diferentes de animales, resultará de gran valor en nuestro intento de iluminar la estructura constitutiva de la socialidad humana.

La paradoja planteada por el desajuste entre el grado de inteligencia atribuible a una cierta especie animal, y el grado de complejidad exhibido por su correspondiente sociedad, se disuelve cuando nos damos cuenta de que esa supuesta paradoja, en realidad, tiene como causa un error categorial. Ese error consiste en la atribución de inteligencia y complejidad a entidades de naturaleza muy diferente. Supóngase que comparamos dos sistemas sociales bien distintos, tales como una colonia de hormigas y una manada de lobos. Como consecuencia de esa comparación, llegamos a la conclusión de que la sociedad de las hormigas parece ser más compleja que la de los lobos, a pesar de que los primeros animales son a todas luces menos inteligentes que los segundos. Se trata de una conclusión de apariencia razonable, pero que ignora un hecho fundamental: el de que las realidades a las que estamos aplicando las nociones de «inteligencia» $\mathrm{y}$ «complejidad», son sujetos constituidos de manera muy distinta en uno y otro caso.

Pues, en efecto, en el caso de la manada de lobos, la realidad a la que atribuimos inteligencia es, de forma muy clara, el animal individual - en principio, cada miembro adulto de la manada. M ientras que en el caso de la colonia de hormigas, por el contrario, la clase de inteligencia que mantiene vivo y viable el correspondiente sistema social no parece residir en los insectos individuales, sino más bien en la colonia como totalidad. Se trata de una suerte de inteligencia colectiva que usaría como recursos la muy limitada inteli gencia individual de los animales involucrados; pero que constituiría un nuevo dominio cognitivo, de naturaleza metaindividual y capaz de multiplicar la eficacia de esos modestos atisbos de inteligencia administrados por los animales individuales. Algo similar sucede cuando atendemos al fenómeno de la complejidad de uno y otro tipo de sociedades. En la colonia de hormigas, es justamente la conducta colectiva la que da muestras de complejidad. La conducta individual de cada hormiga se limita a unas pocas pautas de comportamiento, de carácter fuertemente estereotipado. En la manada de lobos, por el contrario, la complejidad se manifiesta sobre todo en el nivel de la conducta individual los lobos, como los perros, son capaces de desarrollar una cierta «personalidad» que los individualiza y distingue de sus otros congéneres.

\section{Las sociedades animales y sus dos tipos de mapas sociocognitivos: externos e internos}

En las sociedades animales parecen operar, al menos, dos niveles y dos clases de mecanismos sociocognitivos: los que se configuran dentro del individuo social y actúan a través de éste, por un lado, y por otro, los que emergen en el plano de la conducta colectiva y funcionan a escala del sistema social en su conjunto. Conviene precisar, a este respecto, que los mecanismos sociocognitivos a los que me refiero producen en uno y otro caso, como resultado de su acción 
combinada, genuinos mapas (soci o-) cognitivos de carácter dinámico. Estos mapas se producirían y reproducirían en el curso y como resultado de la acción de los individuos, y servirían a su vez para guiar la conducta de éstos. ${ }^{15}$ Son mapas cognitivos societales capaces de determinar, de forma más o menos estricta, los procesos de sincronización conductual que vinculan a los individuos constituyentes de una determinada sociedad - procesos que deben necesariamente producirse para que ésta se constituya y exista como tal. En realidad, una sociedad definiría sus límites, y sería capaz de funcionar como un sistema viable, gracias a la presencia de un conjunto relativamente coherente de tales mapas.

Se trata de mapas dinámicos, porque, por una parte, funcionan como atractores ${ }^{16}$ en relación con las acciones individuales, y, por otra, están siendo constantemente trazados y vueltos a trazar por esas mismas acciones que ellos contribuyen a guiar. Tales mapas fungirían pues a un tiempo como herramientas cognitivas y mecanismos de control, a través de los cuales una particular socie dad se percibe y se organiza a sí misma. Cada especie animal produce espontáneamente mapas sociocognitivos idiosincrásicos, que facultan a esa especie para constituir el estilo de sociedad que le es propio.

En el caso de las sociedades de hormigas, esos mapas son fáciles de identificar, porque tienen una materialización física - 0, más bien, bioquímicadirecta. Están constituidos, principalmente, por los elaborados rastros de feromonas que las hormigas individuales producen sin cesar, a medida que se desplazan por el medio. En ese desplazamiento, las hormigas realizan un repertorio limitado de actividades, que van acompañadas por la producción de las correspondientes clases de feromonas. Los rastros bioquímicos dejados por las hormigas individuales en sus desplazamientos atraen el deambular de otros individuos y provocan en ellos la actividad señalada por la feromona concreta presente en esos rastros. D e esta forma, el movimiento de las hormigas deja de ser azaroso, y tiende a confluir en una red optimizada con vistas a la realización de determinadas tareas. Se trata de un proceso estocástico que permite configurar y dotar de cierta estabilidad a elaboradas redes de sincronización conductual. Y ello sin renunciar a una flexibilidad que posibilita - gracias al mantenimiento del nivel estocástico subyacente- constantes reconfiguraciones parciales - e incluso totales- del sistema, en respuesta a los cambios que experimenta el entorno.

D icho en términos más intuitivos, el rastro de cada hormiga guía el de las que por azar llegan a cruzarse con él, y así el vagar de cada individuo es sólo parcialmente al eatorio, porque resulta atraído por los rastros de los demás - y a su vez atrae potencialmente el deambular de los otros individuos. D e forma que las trayectorias se refuerzan mutuamente y se superponen en un proceso recursivo en el que la conducta individual, y lo que es su producto agregado - la

16. Utilizo el término atractor en su sentido técnico. Véase G LEICK, J. [1988]. 
red de rastros que constituye el mapa sociocognitivo típico de estas sociedades- se realimentan causalmente la una a la otra.

H e señalado la presencia, a lo largo del proceso de la evolución, de dos estrategias básicas seguidas por los animales como caminos alternativos conducentes a las formas más el evadas de complejidad social. Esas dos estrategias, que habrían desarrollado sus peculiares mecanismos sociocognitivos en direcciones opuestas, han sido protagonizadas como dije por los insectos eusociales y por los mamíferos. La primera estrategia, llevada a sus formas más elaboradas por hormigas y termes, ha consistido en el desarrollo de mapas sociocognitivos externos - como los representados por las redes dinámicas de rastros olorosos de feromonas que acabo de describir. Esos mapas existen fuera de los individuos que componen la correspondiente sociedad. Se encuentran materializados directamente en el ecosistema en el que se desenvuelve esa sociedad. Son mapas que tienen un carácter extraneural. Su naturaleza es objetiva: emergen como resultado no intencional, ciego y sin embargo cooperativo, de las interacciones entre los individuos. Son mapas que existen en lo que puede concebirse como un dominio cognitivo metaindividual.

Por una parte, tales mapas se imprimen en el ecosistema natural, y la superposición resultante (mapas + ecosistema natural) constituye lo que podemos denominar el ecosistema social de la correspondiente sociedad. Por otra parte, tales mapas, al ser pragmáticamente interpretados por los individuos sociales, operan como un genuino órgano sociocognitivo. Un órgano que es capaz de computar y controlar tanto las interacciones particulares de los individuos como - a través de ellas- el funcionamiento general de la sociedad en cuestión.

En contraste con esos mapas sociocognitivos externos, de carácter objetivo, extraneural y supraindividual, típicos de las sociedades constituidas por los insectos eusociales, los vertebrados superiores, y en especial los mamíferos, han hecho progresar hasta el virtuosismo unos mecanismos sociocognitivos radicalmente diferentes. Estos últimos animales, en efecto, se han especializado en el desarrollo de mapas soci ocognitivos internos, corporeizados en un sistema nervioso central cada vez más elaborado. Esos mapas son intrínsecos - residen en cada animal - , de manera que tienen una condición individual y subjetiva. Su materialización es de tipo neural, y presumiblemente generan, al menos en las especies más avanzadas, al guna clase de contrapartida mental - en la forma de cierto tipo de imaginería perceptual.

Estos mapas internos, individuales, neurales y, posiblemente, mentales, tienen la facultad de representar dinámicamente, por un lado, amplias porciones del ecosistema natural. Y, por otro, una parte sustancial - e incluso la totalidad- de la sociedad a la que pertenece el individuo que los lleva incorporados en su cerebro, y que los produce y los reproduce, a través del curso de sus interacciones. Los mamíferos superiores, en particular, han desarrollado mecanismos neurales de una sutileza extraordinaria, capaces de elaborar ricas śntesis perceptuales de su medio natural y social. Estos mecanismos permiten la emergencia de complejos universos cognitivos y conductuales, poblados por objetos iden- 
tificables por buen número de características, y cuyas relaciones espaciales y temporales pueden estructurarse con todo detalle.

El córtex cerebral mamífero posee una plasticidad notable, que lo faculta para generar elaboradas síntesis perceptual es y ricos mapas relacionales capaces de asociar objetos particulares a incidencias conductuales singulares. Y esta habilidad presta a los mamíferos superiores un alto grado de flexibilidad en su comportamiento, y una considerable autonomía en sus relaciones con el medio social. M uchos mamíferos superiores, en efecto, son capaces de relacionarse con los congéneres que constituyen ese medio de una manera genuinamente individual, porque pueden distinguir a cada uno de ellos - y pueden ser distinguidos por éstos- como individuos. Este hecho es el que permite al individuo social reconstruir su historia de interacciones con los demás individuos - y, así, adquirir una rudimentaria «memoria biográfica».

Ahora bien, la condición genuinamente individualizada de las relaciones entre los miembros de una sociedad es un instrumento extraordinariamente poderoso de flexibilidad conductual. Y esta plasticidad al nivel micro - muy diferente de la plasticidad al nivel macro, que, como hemos visto, es típica de las colonias de hormigas- inyecta una nueva clase de complejidad en el corres pondiente sistema social, considerado en su conjunto. D e este modo, el desarrollo de mapas cognitivos internos cada vez más complejos abre la posibilidad de que aparezca una forma de creatividad social bien diferente de la que exhiben las sociedades de insectos eusociales. Pues en las indicadas circunstancias pueden emerger configuraciones sociales inéditas, como consecuencia de la adquisición de nuevos rasgos conductuales por parte de los individuos. Sobre la base del desarrollo de tales mapas sociocognitivos internos, es posible incluso el surgimiento de una suerte de protocultura, cuya presencia cabe detectar, por ejemplo, entre ciertas especies no humanas de primates.

\section{Las condición anómala de la socialidad humana}

Llegados a este punto, estamos probablemente en condiciones de enfrentarnos a la pregunta que otorga relevancia sociológica a toda esta discusión acerca de las diferentes estrategias evolutivas que ha seguido el proceso de desarrollo de la socialidad animal. ¿Cuál de esos dos modelos de socialidad - el de los insectos eusociales o el de los mamíferos- resulta más afín a los peculiares mecanismos sociocognitivos que usamos los humanos para edificar nuestros sistemas sociales? Para plantear el interrogante de modo más directo: iestán las sociedades que forma nuestra especie constituidas a la manera de las colonias de hormigas - a través de la acción de mapas sociocognitivos externos (extraneurales, metaindividuales, objetivos), y por tanto no intencionales, ciegos? ¿0 más bien se constituyen al estilo de las sociedades mamíferas - por medio de mapas sociocognitivos internos (neurales, individuales, subjetivos) de carácter intencional?

Es en este momento cuando debemos retornar a la noción de la doble duplicidad de la socialidad humana, bosquejada en las dos primeras secciones de 
este artículo. Porque, en efecto, las preguntas anteriores no admiten una respuesta simple. Por una parte, parece fuera de duda que la socialidad humana, en su nivel más profundo y originario, tiene un carácter inequívocamente mamífero. En realidad, esa socialidad parece ser el resultado del hiperdesarrollo de los mecanismos sociocognitivos en los que se ha ido especializando progresivamente esta clase de animales. ${ }^{17}$ El individuo humano tiene la facultad de producir en su propia mente - en su espacio mental característico, que es la concienciamapas sociocognitivos internos, subjetivos, de una naturaleza extraordinariamente elaborada. Por lo que sabemos, esos mapas son mucho más complejos que los generados, en forma análoga, por otras especies animales. A penas cabe discutir el hecho de que las sociedades humanas se organizan y se controlan - como sistemas de sincronización conductual- primariamente por medio de esos mapas individuales.

En realidad, la socialidad humana, si atendemos nada más al proceso filogenético que le ha dado forma, ${ }^{18}$ tiene un carácter que es lícito calificar de ultramamífero; pues lleva al extremo la tendencia de estos animales a desarrollar mapas sociocognitivos cerebrales con un grado de elaboración cada vez más minucioso. En este sentido, la socialidad humana se opondría diametralmente, por su naturaleza, a la que es propia de los insectos eusociales.

Pero como consecuencia del desarrollo histórico de formas culturales cada vez más complejas - y en particular por efecto de la invención y difusión de nuevos medios de interacción, como la escritura y el dinero- , en las sociedades humanas ha hecho su aparición un nuevo tipo de mapas sociocognitivos. Estos mapas son externos, objetivos y metaindividuales. Son mapas construidos por medio de la cultura material de cada sociedad. Es esta cultura, encarnada físicamente, la que logra transformar de manera cada vez más profunda los variados ecosistemas naturales en los que las sociedades humanas son capaces de prosperar. Y esa transformación convierte el ecosistema natural, que es punto de partida de la acción humana, en un ecosistema profundamente socializado - es decir, en una porción del mundo material que ha sido metamorfoseada por la acción de los individuos que componen una determinada sociedad. Esos mapas sociocognitivos externos, instrumentados por la cultura material humana, resultan extrañamente reminiscentes de los mapas sociocognitivos directamente plasmados en el medio, que, como vimos, son típicos de las sociedades de insectos eusociales.

De manera que la socialidad humana, destinada por un largo proceso evolutivo a adoptar una constitución de tipo ultramamífero, parece volver sobre sus pasos. Y, en el lapso de tiempo - relativamente breve, en la escala de la evolución- de unos pocos milenios, se muestra capaz de desarrollar un clase de mecanismos sociocognitivos que son en cierto modo análogos a los utilizados

17. Los mamíferos forman en efecto una clase dentro del subphylum de los vertebrados, quea su vez pertenece al phylum de los cordados.

18. RicheRSON, P. J. y BOYd, R. [1997]. 
por los insectos eusociales. Así, y de manera enteramente inopinada, la socialidad humana, a partir de cierto momento de su despliegue, parece tender un misterioso puente entre los dos distantes pináculos evolutivos de la socialidad animal.

Este sesgo inesperado, este sorprendente cambio de bando que aparentemente traiciona millones de años de deriva evolutiva, es lo que merece ser considerado como «la anomalía H omo sapiens». ${ }^{19}$ ¿C ómo es posible que sociedades en principio constituidas a partir de un superferolítico desarrollo de la facultad de producir mapas sociocognitivos cerebrales, hayan venido a convertirse en medio privilegiado para el desarrollo de mapas sociocognitivos externos, objetivos, supraindividuales, intencionalmente ciegos? ¿Y cómo es posible que uno y otro tipo de mapas, a pesar de todo, sean capaces de entrar en relación, y puedan producirse y reproducirse los unos a través de los otros?

Pues, en efecto, los mapas sociocognitivos externos encarnados en la cultura material de las sociedades humanas más avanzadas, sólo funcionan a través de su relación con los mapas sociocognitivos internos que los individuos constituyentes de esas sociedades generan, a través de sus cursos de acción y de interacción. De manera que la relación entre uno y otro tipo de mapas no es de mera superposición, sino de coevolución cognitiva y pragmática. ${ }^{20}$ Es así como, con el desarrollo de la civilización, emerge una forma nueva y más compleja de articulación del dominio social humano. U na forma que dará origen a la doble duplicidad que examinamos con anterioridad.

Intentaré reformular del modo más compacto posible el problema que justo ahora comenzamos a atisbar. Lo que podemos concebir como la socialidad primaria de nuestra especie, tiene como mecanismo sociocognitivo básico la constitución y permanente actualización de mapas mentales individuales. Ahora bien, esa «socialidad primaria» se produce y se reproduce - al menos en sociedades provistas de una cultura material lo bastante desarrollada - por medio de su acoplamiento estructural ${ }^{21}$ con lo que llamaremos nuestra socialidad secundaria. Esta «socialidad secundaria» está constituida por mapas cognitivos externos, extramentales y objetivos, materializados en los ecosistemas sociales concretos que son el producto de la desarrollada cultura material que es típica de las sociedades humanas más complejas y recientes. La relación entre esas dos socialidades - 0, si se prefiere, entre sus correspondientes mapas sociocognitivoses de producción y reproducción recíproca, a través de procesos de coevolución

19. No la «anomalía de H omo sapiens». Pues H omo sapiens no es el sujeto de esa anomalía, sino su objeto (recuérdese el célebre artículo de O rtega «El error Berenguer»).

20. Los fenómenos de coevolución son resultado de procesos de transducción ontológica. Es decir, de procesos a través de los cuales dos o más sistemas autoorganizantes, dotados de al guna forma de clausura organizacional, entran en una condición de acoplamiento estructural que les permite traducir sus perturbaciones recíprocas en términos de sus peculiares lógicas organizacionales internas. Véase VARELA, F. J. [1979].

21. En línea con lo sugerido en la nota anterior, hay acoplamiento estructural entre dos o más sistemas cuando los mismos utilizan sus recíprocas perturbaciones para alimentar sus correspondientes lógicas organizacionales internas. 
cognitiva y pragmática posibilitados por esa condición de acoplamiento estructural. Y es esta relación la que se manifiesta, ante la mirada sociológica, a través del fenómeno de la doble duplicidad de la socialidad humana.

\section{Conclusiones: entorno natural socializado, entorno social naturalizado, y vuelta a la doble duplicidad}

Un análisis más detenido de la relación entre las dos socialidades indicadas, y de la mencionada duplicidad, exigiría desarrollos que no caben en los límites de este artículo y habrán de reservarse para otro lugar. M e limitaré aquí a anticipar al gunas de las ideas que deberán vertebrar ese futuro trabajo.

Al hablar de la doble duplicidad de la socialidad humana, señalé que está compuesta de una duplicidad primaria (que se manifestaría en la relación I/me), y una duplicidad secundaria. Ésta última se refiere a la distinción, y a la compleja interacción, entre nuestro self socializado - el cual incluiría la primera duplicidad- y nuestro entorno social objetivo. Un entorno que representa esa «objetividad social» sobre la cual los individuos carecen casi totalmente de control, y que se constituye a partir de procesos sociales de largo al cance, sólo parcialmente intencionales.

Ahora bien, la teoría sociológica suele tener una visión poco clara de ese «entorno social objetivo» cuya presencia - como vimos en la primera sección de este artículo- ha venido advirtiendo desde hace tiempo. Pues rara vez - si alguna - repara en el hecho de que, en realidad, el entorno en cuestión tiene una naturaleza doble. Se compone, por una parte, de lo que voy a llamar el entorno natural socializado. Y, por otra, de lo que denominaré el entorno social naturalizado. El «entorno natural socializado» es ni más ni menos que el ecosistema físico (o, si se prefiere, físico-químico-biológico), en parte artificial, que la cultura material de una determinada sociedad es capaz de producir a partir de cierto medio natural.

El «entorno social naturalizado», por el contrario, tiene un carácter inmaterial y, sin embargo, objetivo. ${ }^{22}$ Es resultado de un hecho ya mentado: en las sociedades humanas más evolucionadas, el desarrollo de una vigorosa cultura material - y especial mente de nuevos y más poderosos medios de interacción, tales como la escritura y el dinero- , está en el origen de un fenómeno peculiar y de gran cal ado. Este fenómeno consiste en lo siguiente: en esas sociedades, algunas de las consecuencias de la acción humana - posiblemente originadas en dominios muy alejados de la vida social - se acumulan, se combinan y se transforman por medio de procesos de reinterpretación intencional protagonizados por diversos agentes. Esas consecuencias, así modificadas, reaparecen ante otros agentes investidas de la condición de circunstancias sociales

22. 0 , si se quiere, exhibe una suerte de materialidad no física. Parece legítimo sostener, en efecto, que existen formas no físicas de materialidad (pensemos, a este respecto, en algunas de las leyes que rigen la evolución de las lenguas, como la de Grimm, 0 en ciertas leyes económicas, como la de G resham). 
objetificadas; de manera que se manifiestan ante ellos como una suerte de entorno social reificado - o, en otros términos, naturalizado- , capaz de cons treñir poderosamente sus opciones como tales agentes.

O bsérvese que ese entorno social naturalizado representa en cierto modo la imagen agencial del entorno natural socializado que acabamos de definir. La relación entre el entorno social naturalizado y el entorno natural socializa do, en efecto, es de mutuo entrañamiento. Y ello en el siguiente sentido: el segundo (el entorno natural socializado) proporciona la base material del primero (el entorno social naturalizado), pues éste no podría constituirse sin la existencia física de aquél; y el primero (el entorno social naturalizado) actúa como la interfaz que acopla la acción humana al segundo (el entorno natural socializado), permitiendo su reproducción y desarrollo material. D e manera que nuestra relación con ese gigantesco y elaboradísimo ecosistema social, en buena parte artificial, que nuestra civilización ha construido sobre todo a lo largo de los últimos siglos, es una relación agencialmente mediada. Es una relación que, para ejecutarse físicamente, debe antes realizarse ${ }^{23}$ agencialmente (es decir, debe prefigurarse en su sentido a través de nuestra acción conciente). ${ }^{24}$ Y esta realización agencial sólo puede concretarse por medio del no menos elaborado sistema de constructos sociales - más o menos reificados- que nuestra cultura espiritual ha venido desarrollando en forma paralela.

En cierto modo, cabe establecer una analogía entre el entorno natural socializado, que ha sido el resultado del impresionante avance de la cultura material humana, y los involuntarios rastros de olor que las hormigas dejan como marca de su actividad - y a través de los cuales, de manera a la vez estocástica y recursiva, organizan esa actividad colectivamente. Como estos rastros, nuestro entorno natural socializado es consecuencia a la vez acumulativa, combinada y recursiva de la actividad de los agentes individuales. És así como se constituye progresivamente en su condición de realidad «objetiva» - en el sentido de que existe con independencia de la intencionalidad de los agentes.

El entorno social naturalizado, por el contrario, no tiene equivalente en el sistema social que forman las hormigas. Pues para que lo tuviera, tendríamos que imaginar una hipotética y peculiar representación (no necesariamente homomórfica), en el sistema nervioso de cada hormiga individual, de parte al menos de esa red de rastros que determina tanto su acción, como la de todas

23. Una cosa es realizar una acción (llegar, a través de un proceso más o menos largo de deliberación, a la decisión de ejecutarla) y otra ejecutarla físicamente. Yo puedo realizar una acción (saludar a alguien) pero ejecutarla mal (porque la persona objeto de mi saludo no consigue verme, o porque en realidad me he equivocado de persona). V éase el capítulo «Acción», en N AVARRO, P. [1994].

24. Utilizo premeditadamente el término conciente, con el objeto de contraponerlo al término consciente. Lo 'conciente' haría referencia a todo lo relacionado con la conciencia, mientras que lo 'consciente' tendría que ver con la mera consciencia. La 'conciencia' es la forma peculiarmente humana de 'consciencia' - es la consciencia autoconsciente. M ientras que ciertos animales sin duda tienen alguna suerte de 'consciencia', sólo el ser humano posee eso que llamamos 'conciencia'. 
sus congéneres. Teniendo en cuenta el formato más bien sencillo y estereotipado de ese sistema nervioso, parece claro que tal representación es imposible.

El aspecto más interesante del asunto es el que atañe a la relación entre esos dos entornos. En cierto modo, el entorno social naturalizado es el resultado de la reflexión, en la conciencia de los agentes, de los efectos sobre la acción humana del entorno natural socializado puesto en pie por la expansión de la cultura material de las sociedades humanas. Ese segundo entorno constituiría el «mapa objetivo» que el primero trataría a su vez de representar. D e alguna forma, el entorno social naturalizado es una suerte de mapa subjetivo del indicado mapa objetivo. A través de ese mapa subjetivo se interpreta pragmáticamente dicho mapa objetivo - y se actúa físicamente sobre él. Todo indica que es esta relación la que permite que se constituya, efectivamente, la célebre pero etérea «mano invisible» de Smith. ${ }^{25}$ Una mano que faculta a las acciones individuales de ciertos agentes para engranar misteriosamente con las acciones individuales de otros - y que permite que esto ocurra sin que medien procesos de interacción reflectiva recíproca entre ellos.

Para terminar, conviene que volvamos sobre la noción con la que comenzábamos el artículo, la idea de la doble duplicidad de la socialidad humana - pues ahora estamos en condiciones de perfilarla con mayor nitidez. La doble duplicidad, según se dijo, articulaba la distinción I/me, que compondría el self, con la distinción entre ese self y el entorno social objetivo. Se presentaba, así, como una duplicidad uno de cuyos miembros encerraba a su vez una duplicidad. Pero ahora vemos que esa duplicidad de segundo nivel es también doble: afecta a los dos miembros de la primera. Porque el segundo miembro de ésta - la objetividad social - encierra asimismo, según se acaba de exponer, una duplicidad generalmente inadvertida: la que hemos señalado mediante la dis tinción entre «entorno social naturalizado» y «entorno natural socializado».

D e manera que la doble duplicidad de la socialidad humana muestra ahora una forma simétrica. La compone, en un primer nivel, la contraposición entre self y entorno social objetivo. $Y$, en un segundo nivel, la duplicidad que habita en cada uno de estos dos miembros componentes de ese primer nivel - respectivamente, la duplicidad I/me y la duplicidad entorno social naturalizado / entorno natural socializado.

\section{Bibliografía}

AleXAN DER, J. C. (1990). Action and Its Environments: Toward a N ew Synthesis, Nueva York, Columbia University Press.

AleXANDER y otros (eds.) (1987). The M icro-M acro Link, Berkeley (CA), University of California Press.

CARABAÑ A, J. (1993). «D e la conveniencia de distinguir entre sociedad y cultura», en E. Lam o de Espin osa yJ. E. Rodríguez Ibáñ ez. (ed.), Problemasde teoría social contemporánea, M adrid, CIS, p. 87-112. 
ChIAlvo, D. R.; M ILlon AS, M . M . [1995]. «H ow Swarms Build Cognitive M aps», en http://www.santafe edusfi/publications/Abstracts/95-03-033abs.html

Colem AN, J. S. (1994). Foundations of Social Theory, Cambridge (M A), Belknap Press ( $\mathrm{H}$ arvard U niversity Press).

D URKH EIM, É. (1987). Las reglas del método sociológico, M adrid, Akal.

Elster, J. (ed.) (1986). Rational Choice, N ueva York, N ew York University Press. GlEICK, J. (1988). Chaos: M aking a N ew Science, N ueva York, Penguin U SA.

GIDDEN S, A. (1986). The Constitution of Society: O utline of the Theory of Structuration, Berkeley (CA), U niversity of California Press.

H IRSCH M AN, A. (1999). Las pasiones y los intereses, Barcelona, Península.

H uber, J. (1991). M acro-M icro Linkages in Sociology, Thousand O aks (CA), Sage Publications.

LAM O DE ESPIN OSA, E. (1996). Sociedades de cultura, sociedades de ciencia, O viedo, Nobel.

Lu h m AN N , N . (1998). Sistemas sociales: lineami entos para una teoría general, Barcelona, Anthropos.

M ARX, K. (1973). El capital. Capítulo VI (inédito), M adrid, Siglo XXI.

M EAD, G. H . (1967). M ind, Self and Society from the Standpoint of a Social Behaviorist, Chicago, University of $\mathrm{C}$ hicago Press.

N AVARRo, P. (1994). El holograma social. U na ontología de la socialidad humana, M adrid, Siglo XXI.

- (1997). «O bjetividad social, subjetividad social, y la noción de complementariedad teórica en sociología», Revista Anthropos, 173/174, julio-octubre de 1997, p. 114125.

- (1999). «Las dos formas de la reflexividad social humana: reflexividad reflectiva y reflexividad disipativa», en R. Ram OS T ORRE y F. García Selgas (eds.), Riesgo, globalización y reflexividad, M adrid, CIS, p. 333-371.

O RTEGA Y G ASSET, J. (1986). I deas y creencias, M adrid, Espasa-C al pe.

Parson S, T. (1964). Social Structure and Personality, N ueva York, Free Press.

PhILLIPS, D . C . (1976). Holistic Thought in Social Science, Palo Alto (CA), Stanford University Press.

RICHERSON, P. J.; BOYD, R. (1997). «T he Evolution of H uman UItra-Sociality», en http://www. sscenet.uda. edu/anthro/faculty/boyd/ultra.pdf

Schellin G, T. C. (1978). M icro M otives and M acro Behavior, N ueva York, W. W. N orton \& Company.

SM ITH, A. (1984). The Theory of M oral Sentiments, Indianapolis (IN ), Liberty Fund, Inc.

- (1991). Wealth of N ations, Amherst (N Y), Prometheus Books.

VAReLA, F. J. (1979). Principles of Biological Autonomy, N ueva York, N orth H olland. WILSON, E. O . (1980). Sociobiología, Barcelona, O mega. 